\title{
Surgical Site Infection by Salmonella Typhimurium Following Breast Cancers Surgery \& Topical Herbal Medicine Use: A Case Report \& Review of the Literature
}

\author{
Suleiman Al-Obeid ${ }^{1^{*}}$, Mohammed Dahman ${ }^{2}$, Suhaib Alothmani ${ }^{2}$, Saud Alrasheedi ${ }^{2}$ and Ziad A Memish ${ }^{3,4,5}$ \\ ${ }^{1}$ Department of Pathology and Laboratory Medicine, Security Forces Hospital, Riyadh, Saudi Arabia \\ ${ }^{2}$ Department of General surgery, Security Forces Hospital, Riyadh, Saudi Arabia \\ ${ }^{3}$ Department of Medicine, College of Medicine, Alfaisal University, Riyadh, Saudi Arabia \\ ${ }^{4}$ Infectious Diseases Division, Prince Mohamed Bin Abdulaziz Hospital, Ministry of Health, Riyadh, Saudi Arabia \\ ${ }^{5}$ Hubert Department of Global Health, Rollins School of Public Health, Emory University, Atlanta, GA, USA
}

${ }^{*}$ Corresponding author: Dr. Suleiman Al-obeid, Department of Pathology and Laboratory Medicine, Security Forces Hospital, Riyadh, Saudi Arabia, E-mail: obeid_jacques@hotmail.com

Received date: March 01, 2018; Accepted date: March 14, 2018; Published date: March 19, 2018

Copyright: @2018 Al-Obeid S, et al. This is an open-access article distributed under the terms of the creative commons attribution license, which permits unrestricted use, distribution, and reproduction in any medium, provided the original author and source are credited.

\begin{abstract}
This is a report of breast abscess due to Salmonella enterica serotype Typhimurium from Saudi Arabia in a 54 years old lady with breast cancer after modified radical mastectomy. Applying contaminated herbal material on the wound could be the main cause of wound infection and localized abscess by Salmonella enterica. Breast abscess or wound infection due to non-typhoidal Salmonella is very rare.
\end{abstract}

Keywords: Breast abscess; Salmonella; Saudi Arabia; Herbal medicine; Surgical site infection; Breast cancer

\section{Background}

Salmonella spp. is known to cause enteric fever, sepsis, and enterocolitis, localized organ disease and could lead to chronic carrier status among infected individuals [1]. If the infection is not treated or proved resistant to treatment, circulating bacteria can infect various organs and cause extra intestinal infection [2]. The pathogenesis of extraintestinal Salmonella infections is affected by many factors: virulence of the strain, host defense mechanisms, and the host's immune response [3]. Risk factors for localized extraintestinal Salmonella infections include: advanced age, gastric surgery, immunosuppressive diseases (HIV infection), immunosuppressive therapy, malignancy (metastatic cancer, lymphoma) and Haemoglobinopathies [4,5]. Breast abscess is commonly caused by Staphylococcus aureus, Streptococcus spp., gram-negative bacilli, and anaerobes [1]. Breast abscess due to non-typhoidal Salmonella are extremely rare [1], but those due to Salmonella enterica serotype Typhi and Paratyphoid have been more frequently reported as a late complication of enteric fever [6].

\section{Case Presentation}

A 45-year-old female patient with stage 3 breast cancer underwent left modified radical mastectomy in March 2017. Postoperatively, the patient was referred to oncology and received 6 cycles of chemotherapy, which ended in August 2017. After a two-week history of discharge from the site of surgery, the patient was referred to our hospital's emergency department and admitted on the $25^{\text {th }}$ of September 2017 with a diagnosis of cancer recurrence vs. abscess. The patient was febrile and had severe pain in the wound site. Clinical examination showed a clear mass that was soft, and sensitive; it was in the left lower area of the closed wound. The patient had no history of gastric enteritis, animal contact, immunological diseases and no positive family history for breast cancer but did have a history of applying topical herbal medication on the site of surgery. MRI of the breast wound showed a large abscess measuring $7.1 \mathrm{~cm}$ anterior to posterior and $6.5 \mathrm{~cm}$ in transverse diameter (Figure 1). Gram staining of the fluid drained and from a wound swab showed numerous polymorph leukocytes and gram-negative bacilli. The material was cultured on $5 \%$ sheep blood and eosin methylene blue (EMB) agar plates and incubated under aerobic and anaerobic conditions at $37^{\circ} \mathrm{C}$. After $24 \mathrm{~h}$. smooth and straight colonies without hemolysis, that were lactose and oxidase-negative, grew on sheep blood and EMB agar. Identification and antibiotic susceptibilities were performed, according to the manufacturer's recommendations, by the microdilution method with commercial dehydrated panels (Dade Behring MicroScan, Sacramento CA, USA) that were read by the Walkaway 96 S1 and interpreted according to CLSI criteria. These colonies were identified as Salmonella enterica serotype Typhi group B1 and the strain was found to be susceptible to ampicillin, ciprofloxacin, trimethoprimSulfamethoxazole, erythromycin, chloramphenicol, ceftazidime, and ceftriaxone. The breast abscess was surgically drained under general anesthesia and the patient was given oral ciprofloxacin $500 \mathrm{mg}$ twice daily. Two weeks later, the wound healed with resolution of the abscess. Ultrasonography after 4 months showed no sign of recurrence of the abscess.

\section{Discussion}

Predisposing factors for breast abscess include: breast malignancy, pregnancy, lactation, hematoma, advanced age, gastric surgery, previous local trauma and drug abuse $[1,4,5]$. Risk of surgical site infection (SSI) is increased in patients undergoing mastectomy and in patients who had an implant or tissue expander placed during surgery. The case reported here had modified radical mastectomy for breast 
Citation: Al-Obeid S, Dahman M, Alothmani S, Alrasheedi S, Memish ZA (2018) Surgical Site Infection by Salmonella Typhimurium Following Breast Cancers Surgery \& Topical Herbal Medicine Use: A Case Report \& Review of the Literature. J Bacteriol Parasitol 9: 336 . doi: $10.4172 / 2155-9597.1000336$

Page 2 of 2

malignancy, and no implant or tissue expander was placed during surgery. Breast abscess are due to Staphylococcus aureus in most of cases; but in our case we report a breast abscess due to Salmonella enterica serotype Typhimurium group B1. When the patient's history was reviewed, it was revealed that the patient had had no episode of gastroenteritis, no contact with animals, and no stool sample was taken before the antibiotic treatment, but there was a history of applying herbal mixtures treatment on the wound. We assumed that applying of herbal medication, which could have been contaminated on the surgical wound, might have contributed to the formation of a $S$. typhimurium wound abscess. In some cases, the patient's breast might have become infected during a transient bacteremic episode originating from the patient's bowel, and the immunosuppressive state of the patient might have facilitated formation of the abscess [5]. The first reported case of breast abscess was by Thayer and Hazen, who isolated Salmonella Typhi from the breast abscess of a young housemaid presenting to the Johns Hopkins Hospital, Baltimore in 1907 [7]. In a literature review, 16 cases of breast abscess due to Salmonella are reported, $75 \%$ due to Salmonella Typhi and 25\% Paratyphoid infection. $80 \%$ of the patients are female and $20 \%$ unknown; the ages are varied between $16-40$ years [8]. In the same study, there are only five cases of breast abscess related to nontyphoidal Salmonella were reported. Four cases are female with age varied from 23 to 66 years old, one female case was unilateral breast cancer and one male cases was unilateral breast abscess with diabetes mellitus type 2 Fernando et al. [8] showed that between 1970 and 2012, less than 15 breast abscess cases due to $S$. Typhi were reported Singh et al. [6] retrospectively evaluated the reported cases of Salmonella breast abscesses in the literature and concluded that there is no a common factor between the patients, but the most of them were between 23 and 45 years of age and, immunocompetent [9]. In this study we present a case report of an infected surgical wound related to non-typhoidal Salmonella. The only evidence concerning the source of infection was the application of possibly contaminated herbal material to the surgical wound.

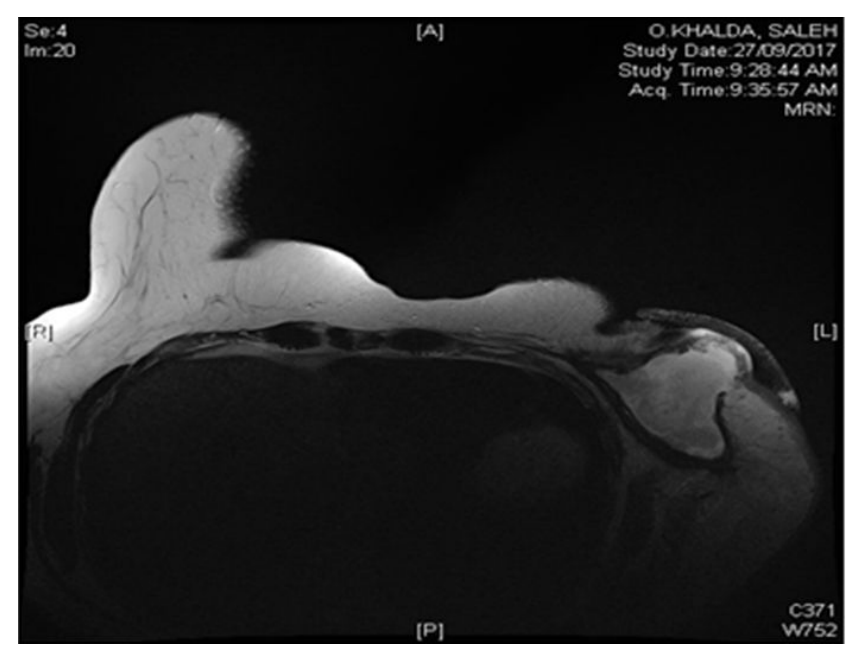

Figure 1: MRI of the closed suction wound.

\section{Conclusions}

Breast abscess after modified radical mastectomy in patients who have had breast cancer are usually due to staphylococcus spp. [1], but rarely can be caused by non-typhoidal Salmonella spp. In the literature several studies revealed the presence of pathogenic bacteria like Salmonella enterica in the polyherbal medicines used for the treatment of different pathology [10]. In our case, we think that applying possibly contaminated herbal mixtures to the wound was the source of infection by Salmonella enterica. Here we advise that, in case of breast surgery, patient need to be educated to avoid applying any herbal medications to the wound. Abscess drainage should be examined in microbiology laboratory for proper diagnosis. In our case the infection was successfully treated with a combination of surgical drainage and antibiotics.

\section{References}

1. Edelstein H (1993) Breast abscess due to Salmonella serogroup B, serotype reading, in a young nonpuerperal woman. Clin Infect Dis 17: 951-952.

2. Fernando S, Molland JG, Gottlieb T (2012) Failure of oral antibiotic therapy, including azithromycin, in the treatment of a recurrent breast abscess caused by Salmonella enterica serotype Paratyphi A. Pathog Glob Health 106: 366-369.

3. Sood S (2015) Breast abscess by Salmonella Paratyphi A: case report and literature review. J Clin Diagn Res 9: 3-4.

4. MacLennan CA (2014) Antibodies and protection against invasive salmonella disease. Front Immunol 5: 635.

5. Brncic N, Gorup L, Strcic M, Abram M, Mustac E (2012) Breast abscess in a man due to Salmonella enterica serotype Enteritidis. J Clin Microbiol 50: 192-193.

6. Jayakumar K, Appalaraju B, Govindan VK (2003) An atypical presentation of Salmonella typhi-a case report. Indian J Med Microbiol 21: 211-212.

7. Thayer WS, Hazen HH (1907) Calcification of the breast following a typhoid abscess. J Exp Med 9: 1-17.

8. Baran I, Aksu N, Aksoy A (2016) Breast abscess due to Salmonella Typhimurium in a patient with rheumatoid arthritis: a case report. BMC Infect Dis 16: 348 .

9. Singh G, Dasgupta M, Gautam V, Behera A, Ray P (2011) Bilateral breast abscesses due to Salmonella Enterica Serotype Typhi. J Glob Infect Dis 3: 402-404.

10. Famewo EB, Clarke AM, Afolayan AJ (2016) Identification of bacterial contaminants in polyherbal medicines used for the treatment of tuberculosis in Amatole District of the Eastern Cape Province, South Africa, using rapid 16S rRNA technique. J Health Popul Nutr 35: 27. 